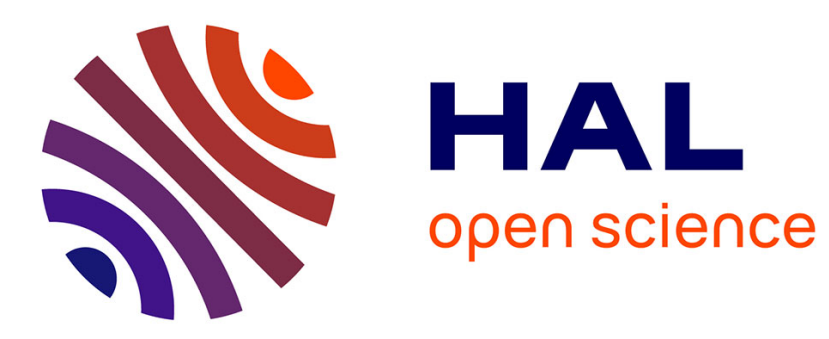

\title{
A robust optimization methodology for preliminary aircraft design
}

Sylvain Prigent, Pierre Maréchal, Aude Rondepierre, Thierry Druot, Matthieu Belleville

\section{- To cite this version:}

Sylvain Prigent, Pierre Maréchal, Aude Rondepierre, Thierry Druot, Matthieu Belleville. A robust optimization methodology for preliminary aircraft design. Engineering Optimization, 2016, 48 (5), pp.883-899. 10.1080/0305215X.2015.1062985 . hal-01493830

\section{HAL Id: hal-01493830 \\ https://hal-univ-tlse2.archives-ouvertes.fr/hal-01493830}

Submitted on 22 Mar 2017

HAL is a multi-disciplinary open access archive for the deposit and dissemination of scientific research documents, whether they are published or not. The documents may come from teaching and research institutions in France or abroad, or from public or private research centers.
L'archive ouverte pluridisciplinaire HAL, est destinée au dépôt et à la diffusion de documents scientifiques de niveau recherche, publiés ou non, émanant des établissements d'enseignement et de recherche français ou étrangers, des laboratoires publics ou privés. 


\title{
A Robust Optimization Methodology for Preliminary Aircraft Design
}

\author{
S. Prigent ${ }^{a b c}$ \\ P. Maréchal ${ }^{b}$ \\ A. Rondepierre ${ }^{b c}$ \\ T. Druot ${ }^{a}$ \\ M. Belleville ${ }^{a *}$
}

\begin{abstract}
This paper focuses on a robust optimization of an aircraft preliminary design under operational constraints. According to engineers know-how, the aircraft preliminary design problem can be modeled as an uncertain optimization problem whose objective (the cost or the fuel consumption) is almost affine, and whose constraints are convex. It is shown that this uncertain optimization problem can be approximated in a conservative manner by an uncertain linear optimization program, which enables the use of the techniques of robust linear programming from Ben-Tal, El Ghaoui, and Nemirovski (2009). This methodology is then applied to two real cases of aircraft design and numerical results are presented.

keywords: Robust linear programming; Optimization under uncertainty; Application to aircraft design.
\end{abstract}

\section{Introduction}

Accounting for uncertainties during the preliminary design phases of an aircraft design is of crucial importance. The objective is to avoid the mistakes appearing during the test phase of the aircraft, which can have a huge impact on the performances. For example when the final mass of the aircraft is assessed, it is important to know the uncertainty that comes with it. Research in this field started around the end of $20^{\text {th }}$ century, with a big amount of work on uncertainty quantification (Bury, 1999; Ellison et al., 2012; Morgan and Henrion, 1990; Duvigneau et al., 2010; Nilsen and Aven, 2003), and on reliable optimization techniques (Geletu et al., 2013; Henrion, 2006; Wendt, Li, and Wozny, 2002; Beyer and Sendhoff, 2007). It is a particular concern at Airbus as attested by the publications (Badufle, 2007; Clément, 2009; Birman, 2013).

Uncertainties on computed solutions must be known precisely and at the very beginning phases of the design in order to have a better knowledge of the solution reliability and thus to control it. However, it raises several problems. Methods have recently been developed in order to obtain more reliable and accurate designs, in reasonable computational time. Among them, the following one can be found: robust design methods (Taguchi, 1978), chance constrained optimization methods using min-max objectives and constraints (Padulo and Liou, 2013; Padulo et al., 2007; Padulo, Campobasso, and Guenov, 2011), sequential methods with reliability assessment (Jaeger, 2013), or surrogate based chance constrained optimization methods (Jin, Du, and Chen, 2003). The main issues are the way of accounting for the uncertainties and the choice of the optimization algorithms. Solving chance constrained programming problem relies on a probability of constraint satisfaction. Robust optimization methods (Beyer and Sendhoff, 2007) have a wide range of applications, with for instance the minimization of the failure probability of a solution or the minimization of the variance of a solution. Here it is proposed to ensure the robustness of the solution whatever the uncertainty, which means that the constraints must be satisfied. This is why the selected method resorts to robust optimization (Ben-Tal, El Ghaoui, and Nemirovski, 2009; Ben-Tal and Nemirovski, 2001).

${ }^{* a}$ AIRBUS Future Project Office, M01 11/1, 316 Route de Bayonne, 31060 Toulouse Cedex 09; ${ }^{b}$ Institut de Mathématiques de Toulouse, University of Toulouse; ${ }^{c}$ INSA Toulouse 
The aircraft preliminary design optimization problem can be written in the following form:

$$
\begin{array}{ll|l}
\min _{X \in \mathbb{R}^{n}} f(X) \quad \text { s.t. } & \begin{array}{l}
g_{i}(X) \leq 0, \forall i=1, \ldots, l \\
\\
X_{\min } \leq X \leq X_{\max } .
\end{array}
\end{array}
$$

In this study, the cost function $f$ can be the fuel consumption over a given mission or the global cost of the aircraft. The optimization variables $X$ can be selected from the following non-exhaustive list: the wing area and the sea-level static thrust for the $2 \mathrm{D}$ case, the wing aspect-ratio, the engine By-Pass ratio, the cruise Mach or the reference cruise altitude for the multidimensional case. The constraint functions $g_{i}$ represent operational constraints, such as the take-off field length, cruise and climb speed ceiling, landing speed, which are known to be convex functions. All these functions and their gradients are provided as black-boxes by SiMCAD, a toolbox developed by Airbus in Scilab. This toolbox is based on low granularity models for geometry, aerodynamics, propulsion, trajectory and masses. More details can be found in (Birman, 2013).

The contribution of this paper is to give a complete methodology for the robust optimization of the aircraft preliminary design. Indeed, all existing studies are mainly deterministic. Until now, uncertainties around the models are taken into account by margins chosen according to engineers know-how, which can sometimes lead to unexpected disappointing performances. Taking uncertainties into account could be done in two ways: chance constrained optimization as done in Prigent et al. (2013), and robust optimization. In this paper is investigated. Due to the particular structure of involved functions, this approach first consists in approximating the initial aircraft preliminary design problem (1) by a linear program and then to apply dedicated robust optimization techniques, namely the ones described in Ben-Tal, El Ghaoui, and Nemirovski (2009).

The outline of the paper is as follows. Section 2 is dedicated to the modeling of the uncertain optimization problem in three steps: first an affine approximation of Problem (1) is built; then uncertainties related to the modeling are defined and the way of dealing with them is presented ; lastly the techniques developed by Ben-Tal, El Ghaoui, and Nemirovski (2009, Chapter 1) are used to obtain an equivalent tractable formulation. Finally, in Section 3, after a short description of the Airbus toolbox SiMCAD, two cases of robust optimization of aircraft design are solved and numerical results are presented.

\section{Acronyms:}

SiMCAD Simple Models for Conceptual Aircraft

COC Cash Operating Cost

SLSTHRUST Sea-Level Static Thrust

MTOW Maximum Take-Off Weight

MZFW Maximum Zero Fuel Weight

TOFL Take-Off Field Length

CLBVZ CLimB Vertical speed ceiling

CRZVZ CRuise Vertical speed ceiling

LDSPEED LanDing Speed

\section{Modeling the uncertain linear optimization problem}

Engineers knowledge and first computations concur in assuming that objective functions such as cost or fuel consumption, are almost affine in the design variables, and that the constraint set could be approximated by a polyhedral set, i.e. by a finite set of affine constraints. This will enable in Section 2.1 to replace Problem (1) by a linear programming problem of the form:

$$
\begin{array}{|cl}
\min _{X \in \mathbb{R}^{n}} & c^{\top} X+d, \\
\text { s.t. } & A \cdot X \leq b, X_{\min } \leq X \leq X_{\max }
\end{array}
$$

where $\mathbf{A} \in \mathbb{R}^{m \times n}, \mathbf{b} \in \mathbb{R}^{m}, \mathbf{c} \in \mathbb{R}^{n}$ and $d \in \mathbb{R}$. The number of constraints is $m+n$. 
The next step will be in Section 2.2 to deal with uncertainties naturally arising in the aircraft modeling. The latter will be expressed as uncertainties on the coefficients $A, b, c$ and $d$. Lastly the uncertain linear optimization problem and its robust counterpart are formulated in Section 2.3 , and it is shown that it could be rewritten as a deterministic linear programming problem.

Note that all functions used along the design process are black-box type functions for which gradients are available.

\subsection{Polyhedral approximation}

Assuming that the objective function $f$ is affine, $f$ can be replaced by its first order approximation at $X_{0}$ :

$$
f(X)=f\left(X_{0}\right)+\nabla f\left(X_{0}\right)^{\top}\left(X-X_{0}\right),
$$

where $X_{0}$ denotes, e.g., the mean point of the bounded domain $\left\{X \in \mathbb{R}^{n}: X_{\min } \leq X \leq X_{\max }\right\}$. Let define:

$$
c=\nabla f\left(X_{0}\right), d=f\left(X_{0}\right)-\nabla f\left(X_{0}\right)^{\top}\left(X-X_{0}\right) .
$$

Let now focus on building a polyhedral approximation of the constraint set $\left\{X \in \mathbb{R}^{n}: g(X) \leq\right.$ $0\}$. For each constraint $g_{j}(X) \leq 0$, the idea is first to compute a family of points $\left(X_{i}^{j}\right)_{i=1, \ldots, m_{j}}$ on the level set $L_{0}\left(g_{j}\right)=\left\{X \in \mathbb{R}^{n}: g_{j}(X)=0\right\}$ and then to build a piecewise linear approximation $\underline{g}_{j}$ of $g_{j}$ :

$$
\underline{g}_{j}(X)=\max \left\{a_{i}^{j \top}\left(X-X_{i}^{j}\right): i=1, \ldots, m_{j}\right\} .
$$

For a given family of points $\left(X_{i}^{j}\right)_{i=1, \ldots, m_{j}}$ on the level set $\mathcal{L}_{0}\left(g_{j}\right)$, two ways for choosing $a_{i}^{j} \in \mathbb{R}^{n}$ are proposed:

- Approximation by tangent hyperplanes. Let define:

$$
a_{i}^{j}=\nabla g_{j}\left(X_{i}^{j}\right), \forall i=1, \ldots, m_{j}
$$

so that $\left\{a_{i}^{j^{\top}}\left(X-X_{i}^{j}\right), i=1, \ldots, m_{j}\right\}$ is a family of hyperplanes tangent to the constraint set $\left\{X \in \mathbb{R}^{n}: g_{j}(X) \leq 0\right\}$.

- Approximation by secant hyperplanes. Assume: $m_{j} \geq n$. For each $n$-uplet $\left(\widetilde{X}_{1}^{j}, \ldots, \widetilde{X}_{n}^{j}\right)$ of points selected among the points $X_{1}^{j}, \ldots, X_{m_{j}}^{j}$, the equation of the hyperplane passing through the points $\tilde{X}_{1}^{j}, \ldots, \widetilde{X}_{n}^{j}$ is of the form:

$$
a^{j \top}\left(X-\tilde{X}_{1}^{j}\right)=0 .
$$

Since the coefficients $a^{j}=\left(a_{i}^{j}\right)_{i=1, \ldots, n}$ are defined up to a multiplicative constant, it is arbitrary imposed: $a^{j \top} \tilde{X}_{1}^{j}=1$, so that $a^{j}$ is solution of the following system: $a^{j \top} \widetilde{X}_{i}^{j}=1$, $i=1, \ldots, n$, i.e.:

$$
\left[\widetilde{X}_{1}^{j}|\ldots| \widetilde{X}_{n}^{j}\right]^{\top} a^{j}=1
$$

In both cases, the constraint $g_{j}(X) \leq 0$ is then replaced by $\underline{g_{j}}(X) \leq 0$. The latter is equivalent to: $a_{i}^{j^{\top}}\left(X-X_{i}^{j}\right) \leq 0$ for all $i=1, \ldots, m_{j}$ and can be rewritten as:

$$
A_{j} X \leq b_{j}
$$

where $A_{j}=\left[a_{1}^{j}|\ldots| a_{m_{j}}^{j}\right]^{\top} \in \mathbb{R}^{m_{j} \times n}$ and $b_{j}=A_{j} X_{i}^{j} \in \mathbb{R}^{m_{j}}$. Lastly the constraints $g_{j}(X) \leq 0$, $j=1, \ldots, l$, are now replaced by the affine constraint:

$$
A X \leq b
$$


where: $A=\left[A_{1}^{\top}|\ldots| A_{l}^{\top}\right]^{\top} \in \mathbb{R}^{m \times n}, b=\left[b_{1}^{\top}|\ldots| b_{l}^{\top}\right]^{\top}$ and $m=\sum_{j=1}^{l} m_{j}$.

In the case where constraints are affine, the tangent hyperplane approximation can be used and is easiest to compute. When the constraint functions $g_{j}, j \in\{1, \ldots, l\}$, are not affine but convex, the points $X_{i}^{j}$ used in the secant hyperplanes computation have to be selected carefully: more precisely, points on the boundary of the domain $\left[X_{\min }, X_{\max }\right]$ have to be chosen in order to obtain a conservative approximation of $\{X \mid g(X) \leq 0\}$. In that case, $g_{j}(X) \leq \underline{g}_{j}(X)$ for all $X$ and all $j=1, \ldots, l$, hence:

$$
\{X \mid g(X) \leq 0\} \subset\{X \mid \underline{g}(X) \leq 0\} .
$$

Let now describe the construction of the points $\left(X_{i}^{j}\right)_{i=1, \ldots, m_{j}}$ for each level set $\mathcal{L}_{0}\left(g_{j}\right), j=$ $1, \ldots, m$. For the sake of clarity the subscript $j$ is omitted in the following explanation. For a given inequality constraint $g(X) \leq 0$, the objective is to find a family of points $\left(X_{i}\right)_{i=1, \ldots, m}$ such that $g\left(X_{i}\right)=0$. This problem is then decomposed into two steps: first computing one point $X_{1}$ in a reasonable computational time, and then from this first point, to compute $X_{2}, \ldots, X_{m}$ such that the family $\left(X_{i}\right)_{i=1, \ldots, m}$ is well-distributed on the level set $\mathcal{L}_{0}(g)$.

\subsubsection{Computing points on the level set $L_{0}(g)$}

In this section the goal is to compute at least one point $X_{1} \in \mathbb{R}^{n}$ satisfying: $g\left(X_{1}\right)=0$. From an analytical point of view, this problem can be formulated as:

$$
\min _{X \in \mathbb{R}^{n}} g(X)^{2}
$$

To solve this problem the use of a gradient-type algorithm (Bonnans et al., 2006) is proposed: let consider an arbitrary point $\tilde{X}_{0} \in \mathbb{R}^{n}$. By definition of the iso-level curve of $g: \tilde{X}_{0} \in \mathcal{L}_{g\left(\tilde{X}_{0}\right)}(g)$. The gradient descent algorithm generates a sequence of iterates $\left(\tilde{X}_{k}\right)$ such that:

$$
\tilde{X}_{k+1}=\tilde{X}_{k}-2 s_{k} g\left(\tilde{X}_{k}\right) \nabla g\left(\tilde{X}_{k}\right),
$$

where $s_{k}>0$ denotes the step length. In this approach the step $s_{k}$ is chosen such that in case of an affine constraint function $g$, Algorithm (5) converges in one single iteration: assuming $g$ is affine, the objective is to compute $s_{k}$ such that: $g\left(X_{k+1}\right)=0$ where $g$ can be expressed as:

$$
g(X)=g\left(\tilde{X}_{k}\right)+\nabla g\left(\tilde{X}_{k}\right)\left(X-\tilde{X}_{k}\right)
$$

This yields:

$$
s_{k}=\frac{1}{2\left\|\nabla g\left(\tilde{X}_{k}\right)\right\|^{2}} .
$$

Finally the following iteration is obtained for the gradient descent method:

$$
\tilde{X}_{k+1}=\tilde{X}_{k}-\frac{g\left(\tilde{X}_{k}\right)}{\left\|\nabla g\left(\tilde{X}_{k}\right)\right\|^{2}} \nabla g\left(\tilde{X}_{k}\right) .
$$

The stopping criterion of the algorithm can be defined by either of the following conditions:

1. $\left|g\left(\tilde{X}_{k}\right)\right|<\varepsilon_{1}$,

2. $\left|s_{k+1}-s_{k}\right|<\varepsilon_{2}$ and $\left.\mid g\left(\tilde{X}_{k+1}\right)-g\left(\tilde{X}_{k}\right)\right) \mid<\varepsilon_{3}$.

In the numerical experiments, $\varepsilon_{1}, \varepsilon_{2}$ and $\varepsilon_{3}$ are set equal to $10^{-3}$. 


\subsubsection{Find the family $\left(X_{i}\right)_{i=1, \ldots, m}$}

Once an initial point $X_{1}$ has been found, the idea is to run through the tangent hyperplane $\left(H_{1}\right)$ : $\nabla g\left(X_{1}\right)^{\top}\left(X-X_{1}\right)=0$ using orthogonal directions. In dimension 2 and 3 a small enough step along these directions (according to a subdivision of the minimum and maximum bounds) is defined. Along each direction a new point is computed from which the previous search algorithm is started. Once the current point goes beyond the bounds of the domain, the direction is no longer used. A well-distributed family of $\left(X_{i}\right)_{i=1, \ldots, m}$ that approximates the level curve $\mathcal{L}_{0}(g)$ is obtained. This technique is heuristic and then improvements are required to develop a better method. Numerical examples in dimensions 2 and 3 works well. In higher dimension it becomes more difficult. A heuristic way could be to first compute a family of points close to the level curve $\mathcal{L}_{0}(g)$ with a low cost, and then to run the previous algorithm from all these points. For example surrogate modeling from the real functions could be used to compute the latter family.

However, a general methodology needs to be developed for a better approximation of the level curve.

\subsection{Modeling Uncertainties}

In the following, uncertainties on coefficients $A, b, c$ and $d$ are supposed to be known. In practice, they can be provided by engineers, or obtained by uncertainty propagation methods. The linear programming problem taking into account the uncertain coefficients $A, b, c, d$ can then be defined. The following family of optimization problems is obtained:

$$
\begin{array}{cl}
\min _{X \in \mathbb{R}^{n}} & c^{\top} X+d, \\
\text { s.t. } & A \cdot X \leq b, \quad X_{\text {min }} \leq X \leq X_{\max }
\end{array}
$$

where $(A, b, c, d) \in \mathcal{U}$ and $\mathcal{U} \subset \mathbb{R}^{m \times n} \times \mathbb{R}^{m} \times \mathbb{R}^{n} \times \mathbb{R}$ is the uncertain set. Let state:

$$
D=\left[\begin{array}{ll}
c^{\top} & d \\
A & b
\end{array}\right], D \in \mathbb{R}^{(1+m) \times(n+1)} .
$$

In order to remain consistent with the analysis of A. Ben-Tal, L. El Ghaoui and A. Nemirovski in Ben-Tal, El Ghaoui, and Nemirovski (2009), it is assumed that $\mathcal{U}$ admits an affine parametrization such that:

$$
\mathcal{U}=\left\{D=\left[\begin{array}{cc}
c^{\top} & d \\
A & b
\end{array}\right] \in \mathbb{R}^{(1+m) \times(n+1)} \mid D=D^{0}+\sum_{k=1}^{L} \zeta_{k} D^{k}, \text { with } \zeta \in \mathcal{Z} \subset \mathbb{R}^{L}\right\},
$$

where $\mathcal{Z} \subset \mathbb{R}^{L}$ denotes the perturbation set, $D^{0}$ is the nominal value, and $D^{k}$ is the variation.

In a first approach the worst case situation is considered, in which: $L=(m+1)(n+1)$ and the variations $D^{k}$ are defined such that:

$$
D_{i, j}^{k}= \begin{cases}1 & \text { if } l=(n+1)(i-1)+j, \\ 0 & \text { otherwise. }\end{cases}
$$

The perturbation set $\mathcal{Z}$ is then of the form:

$$
\mathcal{Z}=\bigotimes_{k=1}^{L}\left[\zeta_{k}^{\text {inf }}, \zeta_{k}^{\text {sup }}\right]
$$

The definition of $\mathcal{Z}$ from (11) is a worst case approximation of the uncertainty set in the sense that the uncertain set is probably overestimated. This overestimation must be dealt with cautiously so that it does not induce infeasibility of the problem.

Note that the definition of the uncertainty set $\mathcal{U}$ can be simplified by introducing the affine transformation $\rho_{k}$ :

$$
\rho_{k}:[-1,1] \rightarrow\left[\zeta_{k}^{\text {inf }}, \zeta_{k}^{\text {sup }}\right]
$$




$$
\xi \mapsto \frac{\zeta_{k}^{\text {sup }}-\zeta_{k}^{\text {inf }}}{2} \xi+\frac{\zeta_{k}^{\text {sup }}+\zeta_{k}^{\text {inf }}}{2} .
$$

The uncertainty set $\mathcal{U}$ can be rewritten as:

$$
\begin{aligned}
\mathcal{U} & =\left\{D=\left(D^{0}+\sum_{k=1}^{L} \frac{\zeta_{k}^{\text {sup }}+\zeta_{k}^{\text {inf }}}{2} D^{k}\right)+\sum_{k=1}^{L} \frac{\zeta_{k}^{\text {sup }}-\zeta_{k}^{\text {inf }}}{2} \xi_{k} D^{k} \mid \xi \in[-1,1]^{L}\right\}, \\
& =\left\{D=\hat{D}^{0}+\sum_{k=1}^{L} \xi_{k} \hat{D}^{k} \mid \xi \in[-1,1]^{L}\right\}
\end{aligned}
$$

in which

$$
\hat{D}^{0}=D^{0}+\sum_{k=1}^{L} \frac{\zeta_{k}^{\text {sup }}+\zeta_{k}^{\text {inf }}}{2} D^{k}, \quad \hat{D}^{k}=\frac{\zeta_{k}^{\text {sup }}-\zeta_{k}^{\text {inf }}}{2} D^{k} .
$$

\subsection{The uncertain linear optimization problem and its robust counter- part}

Following Ben-Tal, El Ghaoui, and Nemirovski (2009), the Problem (7) is equivalently reformulated in such a way that uncertainties only appear in the constraints:

$$
\begin{array}{c|l}
\min _{(X, t) \in \mathbb{R}^{n+1}} t \text { s.t. } & \begin{array}{l}
A X \leq b, \\
\end{array} \\
c^{\top} X+d \leq t, X_{\min } \leq X \leq X_{\max }
\end{array}
$$

for any $(A, b, c, d) \in \mathcal{U}$. By definition, a robust solution of (17) is an optimal solution of its robust counterpart and the robust counterpart of the Problem (17) is defined by:

$$
\min _{(X, t) \in \mathbb{R}^{n+1}}\left(\begin{array}{l|l}
\sup _{(A, b, c, d) \in \mathcal{U}} t
\end{array}\right) \text { s.t. } \mid \begin{aligned}
& A X \leq b, c^{\top} X-t \leq-d, \\
& X_{\min } \leq X \leq X_{\max }, \\
& \forall(c, d, A, b) \in \mathcal{U},
\end{aligned}
$$

which is equivalent to:

$$
\begin{array}{l|l}
\min _{(X, t) \in \mathbb{R}^{n+1}} t \text { s.t. } & \begin{array}{l}
A X \leq b, c^{\top} X-t \leq-d \\
X_{\min } \leq X \leq X_{\max } \\
\forall(c, d, A, b) \in \mathcal{U}
\end{array}
\end{array}
$$

Let $A:=\left[a_{1}^{\top}, \ldots, a_{m}^{\top}\right]^{\top}$. The robust counterpart (18) can be rewritten as:

$$
\begin{array}{l|l}
\min _{(X, t) \in \mathbb{R}^{n+1}} t \text { s.t. } & \begin{array}{l}
a_{i}^{\top} X \leq b_{i}, i=1, \ldots, m \\
c^{\top} X-t \leq-d \\
X_{\min } \leq X \leq X_{\max } \\
\forall(c, d, A, b) \in \mathcal{U} .
\end{array}
\end{array}
$$

By means of the machinery of Ben-Tal, El Ghaoui, and Nemirovski (2009), the latter can be rewritten into the following deterministic linear optimization problem (see appendix A for technical details):

$$
\begin{array}{l|l}
\min _{\tilde{X} \in \mathbb{R}^{2 n+1}} C^{\prime \top} \tilde{X} \text { s.t. } & \begin{array}{l}
A \tilde{X} \leq b \\
X_{\min } \leq X \leq X_{\max }
\end{array}
\end{array}
$$

where the optimization variables are: $\tilde{\mathbf{X}}=\left(X^{\top}, t, \mathbf{u}^{\top}\right)^{\top}$ and:

$$
\mathbf{C}^{\prime}=(0, \ldots, 0,1,0, \ldots, 0)^{\top} \in \mathbb{R}^{2 n+1},
$$




$$
\begin{aligned}
\mathbf{A} & =\left(\begin{array}{ccc}
I_{n} & 0_{n} & -I_{n} \\
-I_{n} & 0_{n} & I_{n} \\
\hat{A^{(0)}} & 0_{m} & P^{(a)} \\
\hat{\left.\mathbf{C}^{(0)}\right)^{\top}} & -1 & \mathbf{P}^{(\mathbf{c})}
\end{array}\right) \in \mathbb{R}^{(2 n+m+1) \times(2 n+1)}, \\
\mathbf{b} & =\left(0_{1 \times 2 n}, \hat{\mathbf{b}}^{(\mathbf{0})}-\mathbf{P}^{(\mathbf{b})},-P^{(d)}-\hat{d}^{(0)}\right)^{\top} \in \mathbb{R}^{(2 n+m+1)}
\end{aligned}
$$

The scalars, vectors and matrices $\hat{A^{(0)}}, \hat{\mathbf{C}^{(\mathbf{0})}}, P^{(a)}, \mathbf{P}^{(\mathbf{c})}, P^{(b)}$ and $P^{(d)}$ are defined in appendix A. Finally a linear program with $(2 n+1)$ unknowns and $(2 n+m+1)$ constraints, where $m$ is the number of initial constraints, is obtained. For example, in the 2-dimensional case-study, it yields that $X \in \mathbb{R}^{2}, \mathbf{u} \in \mathbb{R}^{2}$ and $t \in \mathbb{R}$.

\section{Application: Aircraft Preliminary Design Robust Opti- mization}

Preliminary aircraft design is the first step in the passenger transport aircraft design process (Badufle, 2007). The goal is to choose, among several concepts, the one which would be relevant according to a well defined objective (cost, fuel consumption or environmental impact). For each concept, the main aircraft parameters have to be assessed consistently according to a common set of requirements.

In this section the aircraft preliminary design process is first briefly presented and the related uncertain optimization problem is defined. The way to propagate uncertainties through the models is then described, and finally the whole methodology is applied to two real cases of aircraft design.

\subsection{The Aircraft Preliminary Design Process}

The design process is multidisciplinary since several physics such as geometry, aerodynamics, mass, propulsion, performance and cost analysis are involved.

The used aircraft toolbox is called SiMCAD (Simple Models for Conceptual Aircraft Design), developed in Scilab (Scilab Enterprises, 2012) and dedicated to research activity in the domain of Overall Aircraft Design. It is a toolbox of models that allows to run simple, but realistic, pre-design processes involving all main physics of an aircraft. It offers the possibility to test multi-disciplinary, multi-level, multi-objective and robust optimization strategies without having to manage huge amount of data and huge computation time. A complete description of these models can be found in (Birman, 2013).

More precisely, semi-empirical models are used for the aerodynamics and the masses, and simplified physical models of operational performances. To sum up, all combined models count around 180 parameters and 50 functions. They have been validated on existing aircrafts. Some optimization parameters that will allow to define an aircraft configuration have now to be chosen. Engineers practice and know-how lead to choose the Wing Area that controls the dimension of the wing and the Sea Level Static Thrust (SLSThrust) that controls the engine size.

Now, the aircraft configuration has to be optimized according to a given objective. It is here chosen the Cash Operating Cost (COC) which takes into account the fuel, the masses, the engine and airframe maintenance, the crew cost and some navigation charges. Some requirements are also selected such as the range of the aircraft, that correspond to the customer demands (see Table 1). Operational needs for safety and operations define a set of inequality constraints described in Table 2.

Models and functions that drive the aircraft configuration computation, are sequential. The whole process can be represented from a global point of view by the diagram presented in Figure 1: it shows the way the different physics interact, how an aircraft is computed from requirements and input variables and how objectives and constraints are computed. 
Table 1: Description of the baseline aircraft requirements.

\begin{tabular}{ll}
\hline Name & Value \\
\hline Number of Passengers (Npax) & 180 \\
Design Range & $2000 \mathrm{NM}$ \\
Cruise Mach number & 0.65 \\
Wing Aspect Ratio & 12 \\
Number of Turbofan Engine & 2 \\
Engine By Pass Ratio & 10 \\
Top of Climb altitude & $35000 \mathrm{ft}$ \\
Engine Overall Pressure Ratio & 40 \\
\hline
\end{tabular}

Table 2: Objective and Constraints.

\begin{tabular}{llcll}
\hline Objective & $f$ & Cash Operating Cost (COC) & $\$ /$ trip \\
\hline & $g_{1}$ & Take-off field length 1 (at Sea Level) (TOFL1) & $\leq$ & $2000 \mathrm{~m}$ \\
& $g_{2}$ & Take-off field length 2 (in High \& Hot conditions) (TOFL2) & $\leq$ & $2500 \mathrm{~m}$ \\
Constraints & $g_{3}$ & Climb vertical speed ceiling (CLBVZ) & $\geq$ & $500 \mathrm{ft} / \mathrm{min}$ \\
& $g_{4}$ & Cruise vertical speed ceiling (CRZVZ) & $\geq$ & $300 \mathrm{ft} / \mathrm{min}$ \\
& $g_{5}$ & Landing Speed (LDSPEED) & $\leq$ & $130 \mathrm{kt}$ \\
\hline
\end{tabular}

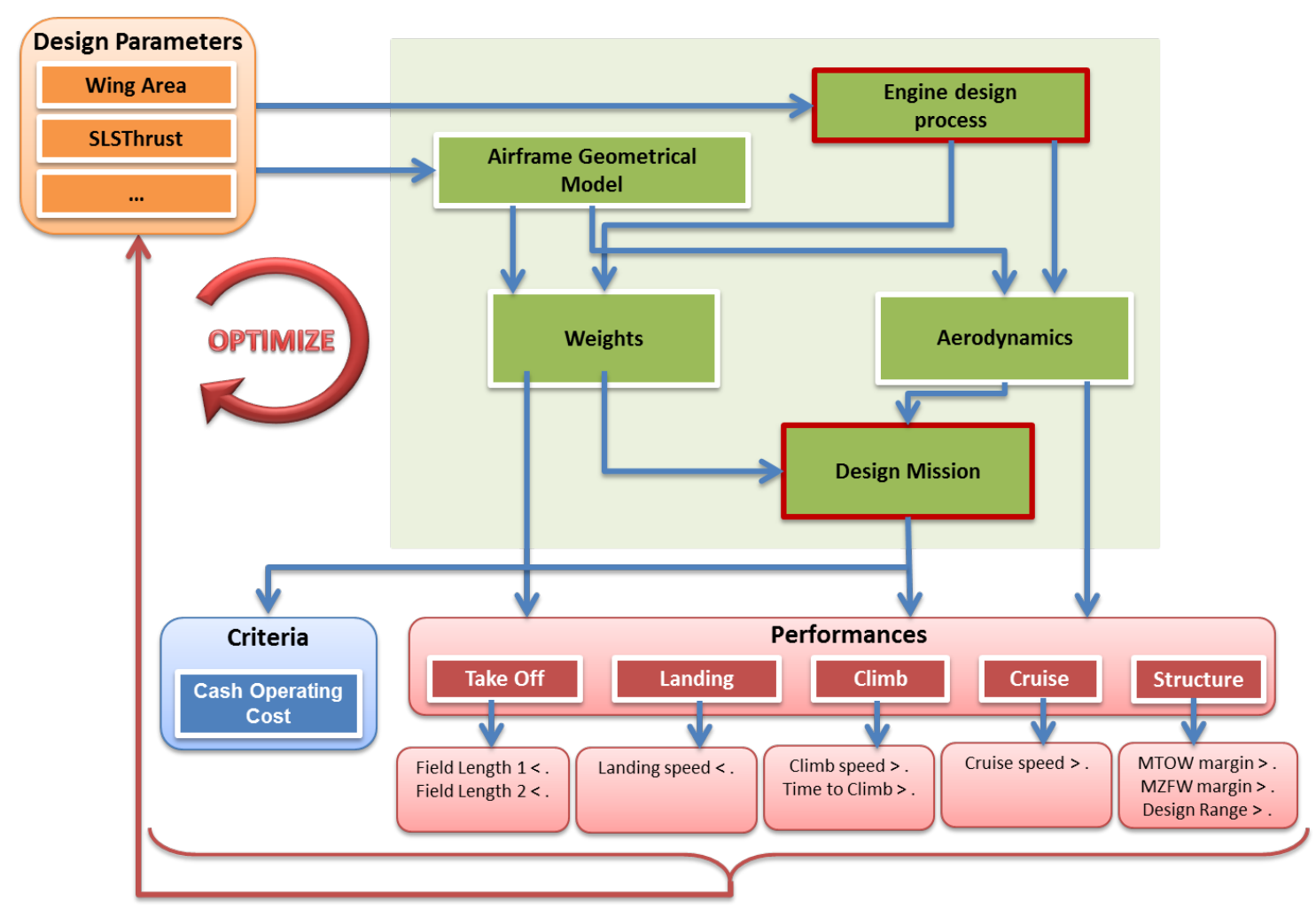

Figure 1: Aircraft Simple Design Process Diagram.

Moreover, the design process is significantly improved when the uncertainty associated to each criteria is provided. Therefore robust optimization has a high added value compared to a classic deterministic one. As mentioned before, uncertainty quantification has already been assessed 
and the most important impact of uncertainty is coming from the computation of aerodynamic efficiency, specific fuel consumption and structural weight. Taking these uncertainties into account, it is presented in this section a two-dimensional and a three-dimensional application cases. The different steps of the approach are summarized hereafter:

- Build an affine approximation for the objective and a polyhedral approximation for the constraints set.

- Quantify and propagate uncertainties with a first order approximation method in order to obtain $L^{\infty}$ boxes on all $\zeta^{\prime}$ s.

- Build corresponding $A, b, c$ and $d$ in order to obtain the Problem (20)

- Solve the linear constrained programming problem (20) using the simplex algorithm (Karmarkar, 1984). Here the linpro function from Scilab is used.

In each test case, the robust solution, i.e. the solution of (20), is compared to the (non robust) solution of the initial deterministic optimization problem (1). The latter is solved using a Feasible Sequential Quadratic Programming algorithm (Zhou and Tits, 1992), here the fsqp Scilab function.

\subsection{Uncertainty propagation method}

The processes involved in the preliminary aircraft design, are bringing uncertainties. They are based on semi-empirical models in which some equations are replaced by regressions from databases. Recently, these uncertainties have been quantified (Birman, 2013). It is now the time to take them into account in the design process.

To evaluate uncertainties on the coefficients $A, b, c, d$ of the Problem (2), several propagating methods can be used (Padulo and Liou, 2013; Padulo et al., 2007; Padulo, Campobasso, and Guenov, 2011; Prigent et al., 2013). The Monte-Carlo sampling is the more accurate one but also the one with the highest computational cost. Moreover the following important information has to be taken into account : the evaluation of the constraints and the objective functions is computationally expensive and the constraints and the objective functions are almost affine.

This is why a moment based propagation method with a first order Taylor approximation of the uncertain function, is used: it is a non intrusive method which offers in this case a good compromise between accuracy and computational cost. The main steps of the propagation method are the following:

- Get or compute the first moments of the model input uncertainty distributions,

- Propagate moments through computation process and get the moments of the output uncertain values using a first order moment method (Lee and Kwak, 1987; Tichỳ, 1994),

- Use these moments to determine the uncertainty set on coefficients $A, b, c$ and $d$. This is done by following Moments Methods from Pearson (1936), that allows to reconstruct a distribution from its moments. Based on this approach, Birman (2013) shows that distributions parameters can be computed as a function of the moments and thus determine the distribution support. The bounds of the supports are then the interval of output uncertainty.

An other example of use of the moment propagation method can be found in Prigent et al. (2013).

\subsection{Robust Aircraft Design Optimization}

The baseline short range aircraft presented in Section 3.1 is chosen. The corresponding robust optimization problem is defined by:

$$
\min _{X \in \mathbb{R}^{n}} f(X) \text { s.t. } \mid \begin{aligned}
& g_{i}(X) \leq 0, \quad i=1, \ldots, 5, \\
& X_{\min } \leq X \leq X_{\max },
\end{aligned}
$$


where the objective and the constraints are defined in Table 2. All constraints are written under the standard form: $g_{i}(X) \leq 0$, and the optimization variables are defined in Table 3. They have to satisfy box constraints. In the case $n=2$ the cruise Mach is set to 0.65 .

Table 3: Optimization variables $\left(x_{1}, x_{2}\right)$ when $n=2$ and $\left(x_{1}, x_{2}, x_{3}\right)$ when $n=3$.

\begin{tabular}{lccc}
\hline & Name & Unit & Lower and Upper bounds \\
\hline$x_{1}$ & Wing Area & $m^{2}$ & {$[100,170]$} \\
$x_{2}$ & Sea-Level Static Thrust & daN & {$[9000,13000]$} \\
$x_{3}$ & Cruise Mach & mach & {$[0.65,0.76]$} \\
\hline
\end{tabular}

In what follows, the objective function $f$ denotes the Cash Operating Cost (COC) computed with respect to the Wing Area $x_{1}$, the Sea-Level Static Thrust $x_{2}$ and the Cruise Mach $x_{3}$. This function is almost affine in $\left(x_{1}, x_{2}, x_{3}\right)$ while the constraint functions $g_{i}$ are convex.

\subsubsection{Case $n=2$}

In two dimensions, the formulation of the problem is the following one:

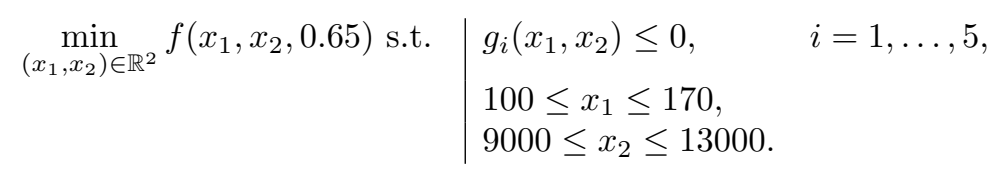

For a better understanding of the proposed approach, Figure 2 shows a 2D map with constraints and some level curves of the objective function.

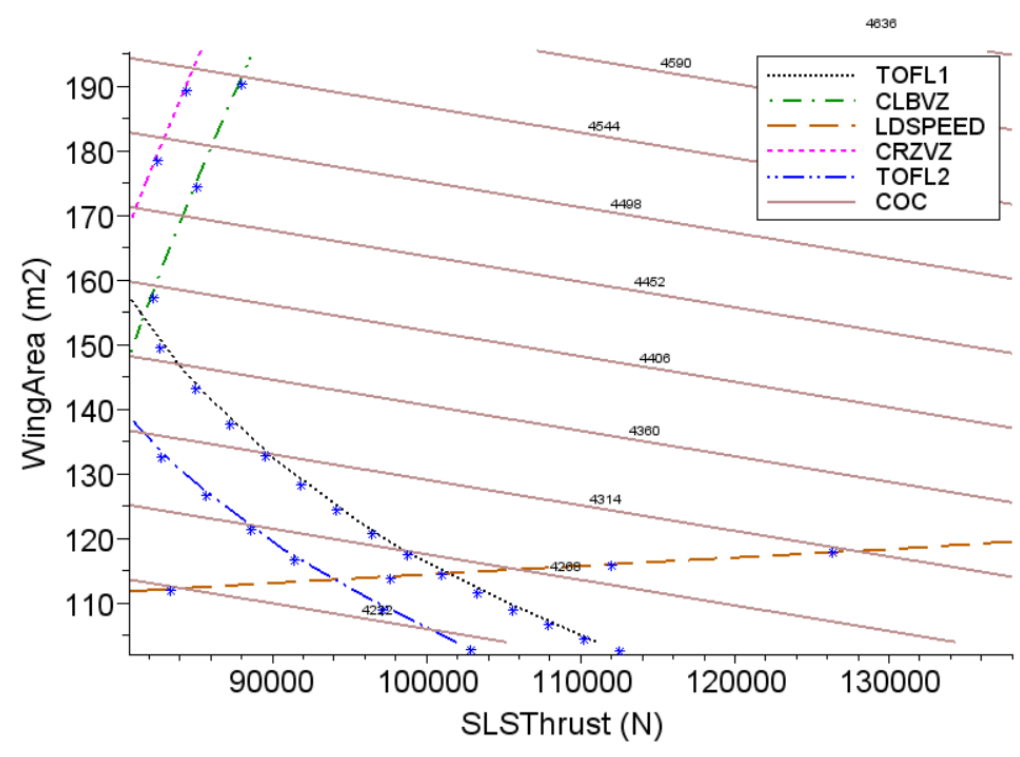

Figure 2: Aircraft 2D Design Problem, with constraints and objective level curves.

The first step of this approach is to compute the affine approximation of $f$ at the mean point of the domain of definition, and the piecewise affine approximation of the $g_{i}$ at points $X_{j}^{i}$, $j=1, \ldots, m_{j}$. For this the method to find points on each constraint level curve is applied. These points are the crosses from Figure 2. They will also be represented in the next figures. Then, as constraints are convex functions, the secant hyperplanes are built. Observe that the more nonaffine the constraint, the higher the number of approximating hyperplanes. Figure 3 shows the 
polyhedral approximation of the constraints set and some level curves of the linearized objective. The corresponding hyperplanes equations are of the form:

$$
a_{1}^{(i)} x_{1}+a_{2}^{(i)} x_{2}=1, i=1, \ldots, m .
$$

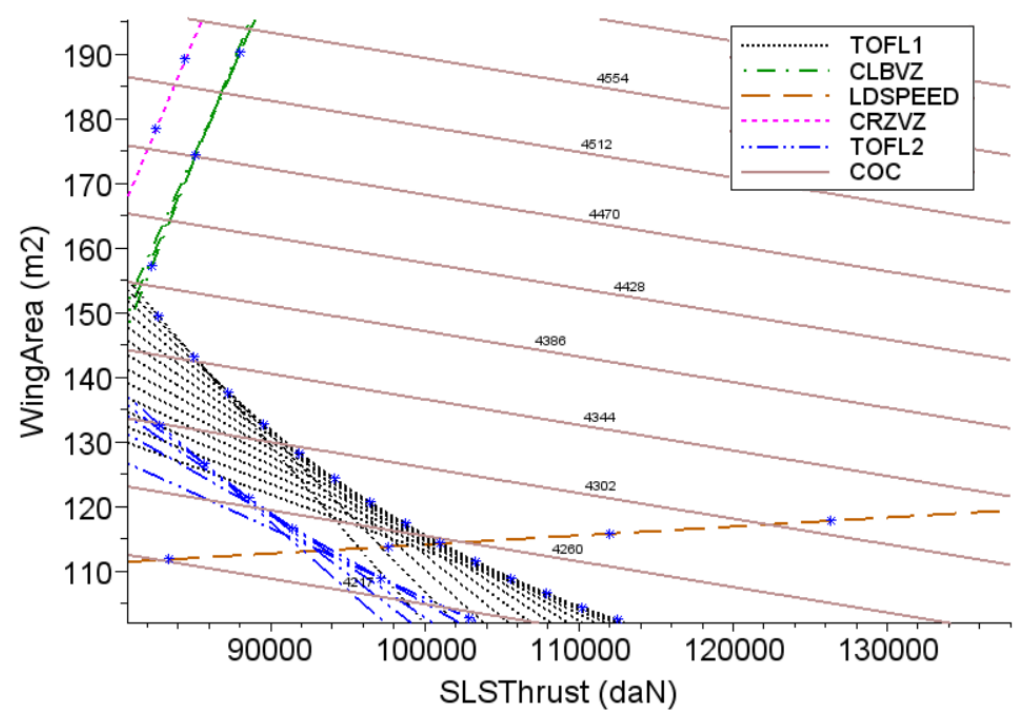

Figure 3: Approximation of Aircraft 2D Design Problem, with piecewise affine constraints and affine objective.

Once the equations of the constraints and of the objective are obtained, the next step is the propagation of uncertainty. The following sources of uncertainty are considered:

- from aerodynamic models: Lift over Drag coefficient (LoD) is calculated with an error of $\pm 1 \%$ around its nominal value,

- from mass models: Maximum Overall Empty Weight (MWE) is calculated with an error of $\pm 1 \%$ around its nominal value,

- from propulsion models: Specific Fuel Consumption (SFC) is calculated with an error of $\pm 1 \%$ around its nominal value.

The first order Taylor based propagation method gives as a result the uncertain interval on $\left(a_{1}^{(i)}, a_{2}^{(i)}\right), i=1, \ldots, m$. The uncertainty interval is also computed for the affine objective function coefficients.

Finally the original problem is reformulated as a linear programming problem such as (20). In this case, with an initial number of approximating hyperplanes $m=25$, it yields a linear programming problem in dimension 5 with a total of 30 constraints. Using a classical linear programming solver (linpro from scilab) based on the simplex method the obtained solution is presented in Figure 4. Constraints at the solution point are also represented on this figure. And the solution is:

$$
X_{\text {sol }}=\left(10292 \mathrm{daN}, 115.6 \mathrm{~m}^{2}\right) \text {, with the objective value: } C O C=4285 \$ / \text { pax. }
$$

The robust solution $X_{\text {sol }}$ is then compared to the non robust solution $X_{\text {sol }}^{\text {det }}$ of the initial deterministic optimization problem (1):

$$
X_{\text {sol }}^{\text {det }}=\left(10121 \mathrm{daN}, 114.3 \mathrm{~m}^{2}\right) \text {, with the objective value: } C O C^{\text {det }}=4279 \$ / \text { pax. }
$$




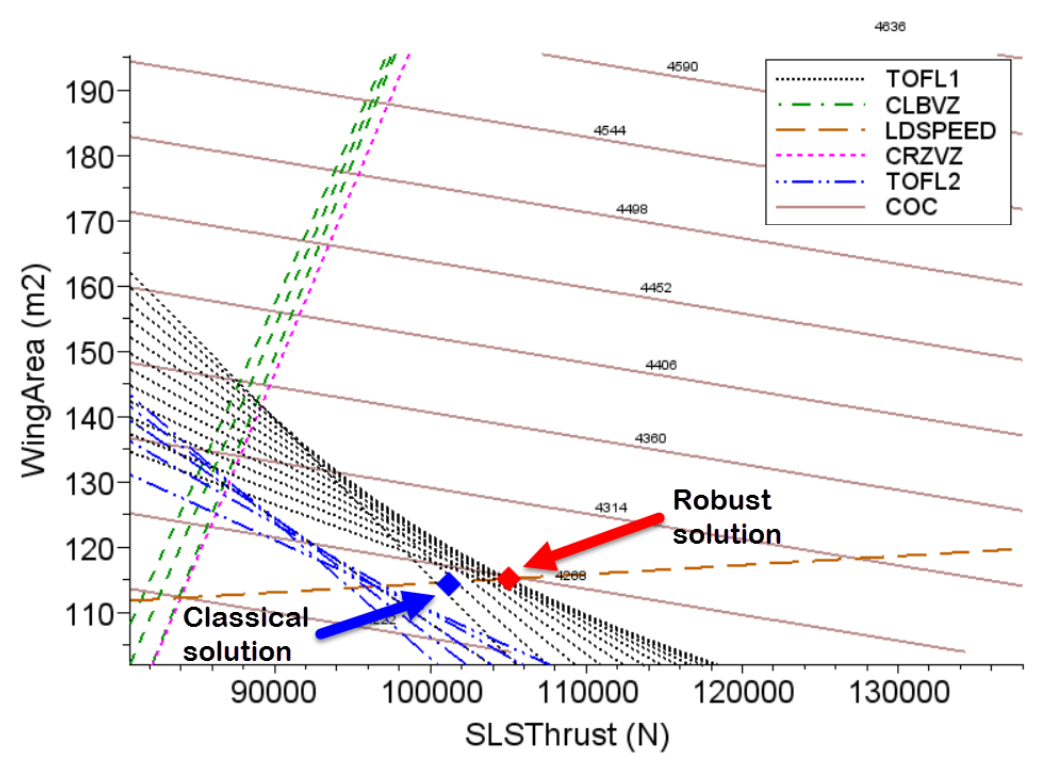

Figure 4: Robust Approximation of Aircraft 2D Design Problem and its solution.

Table 4: Constraints reliability assessment at point $X_{\text {sol }}$ (case $\left.n=2\right)$.

\begin{tabular}{lcc}
\hline Constraint & Upper Bound $\left(g^{\max }\left(X_{\text {sol }}\right)\right)$ & Relative Upper Bound \\
\hline Take-off field length 1 & -5.8 & -0.003 \\
Take-off field length 2 & -176 & -0.07 \\
Climb vertical speed ceiling & -3 & -1.2 \\
Cruise vertical speed ceiling & -3.1 & -2.0 \\
Landing Speed & -0.02 & -0.0003 \\
\hline
\end{tabular}

As expected, the price of robustness is a small increase of cash operating cost $(\mathrm{COC})$ value.

The last step is to verify the robustness of the solution $X_{\text {sol }}$. For that a reliability assessment of the obtained solution point $X_{\text {sol }}$ is done with the original constraint functions. Using the previously mentioned propagation method the interval of error on each constraint is computed (written in the form $g(X) \leq 0)$. For a constraint $g\left(X_{\text {sol }}\right)$ the uncertain interval $\left[g^{\min }\left(X_{\text {sol }}\right), g^{\max }\left(X_{\text {sol }}\right)\right]$ is obtained. Then the solution is robust, meaning that constraints are always satisfied, if and only if $g^{\max }\left(X_{\text {sol }}\right) \leq 0$. Results are in Table 4. Relative upper bound represents the upper bound divided by the required value of Table 2 for each constraint. It is observed that active constraints (Take-off field length 1 and Landing Speed) have the lowest relative reliability margin as expected.

\subsubsection{Case $n=3$}

In this case, the cruise Mach number is added to the optimization variables. The formulation of the problem is the following one:

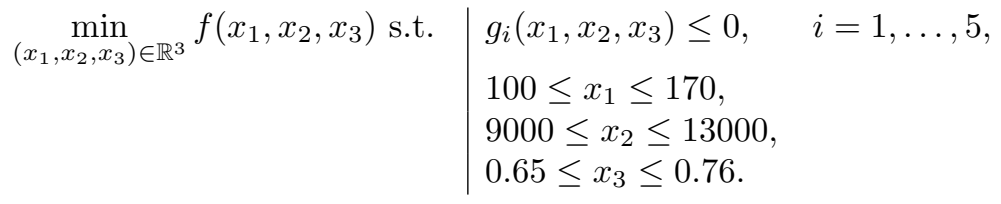

The same steps as for the case $n=2$ are followed. An affine approximation of $f$ at the mean point of the domain of definition is first computed, and then a piecewise affine approximation of the $g_{i}$ 
at points $X_{j}^{i}, j=1, \ldots, m_{j}$. For that the method to find points on each constraint level curve is applied. Since the constraint functions are known to be convex, secant hyperplanes are built. Then the same sources of uncertainties from aerodynamic, mass and propulsion models are considered. Finally the problem is reformulated in order to obtain a linear programming formulation such as in Equation (20). In this case, with an initial number of approximating hyperplanes $m=43$, it yields a linear programming problem in dimension 7 with a total of 50 constraints. Using the linpro linear programming solver from Scilab the solution is:

$$
X_{\text {sol }}=\left(9764 \mathrm{daN}, 141.2 \mathrm{~m}^{2}, \text { mach 0.76), with: } C O C=4080 \$ /\right. \text { pax. }
$$

The robust solution $X_{\text {sol }}$ is then compared to the non robust solution $X_{\text {sol }}^{\text {det }}$ of the initial deterministic optimization problem (1):

$$
X_{\text {sol }}^{\text {det }}=\left(9306 \mathrm{daN}, 137 \mathrm{~m}^{2} \text {, mach 0.76), with: } C O C^{\text {det }}=4070 \$ /\right. \text { pax. }
$$

The reliability assessment at the solution is also done. Table 5 presents each constraint. Reliability is satisfied. Let finally have an insight into the computational cost of the robust design approach:

Table 5: Constraints reliability assessment at point $X_{\text {sol }}$ (case $\left.n=3\right)$.

\begin{tabular}{lcc}
\hline Constraint & Upper Bound & Relative Upper Bound \\
\hline Take-off field length 1 & -95 & -0.05 \\
Take-off field length 2 & -267 & -0.11 \\
Climb vertical speed ceiling & -1.1 & -0.44 \\
Cruise vertical speed ceiling & -1.2 & -0.8 \\
Landing Speed & -0.41 & -0.006 \\
\hline
\end{tabular}

Table 6 presents the number of calls to the process in the deterministic case and in the robust case. Note that the computational effort spent using the robust approach, comes only from the linearization process.

\section{Conclusion and perspectives}

This paper proposes a methodology to apply techniques of robust linear programming to the aircraft preliminary design problem. Under the assumptions of an affine objective and convex constraints, the problem is first approximated in a conservative manner by an uncertain linear program. In the case when the uncertainty set on the output is unknown, a propagation method can be used. The uncertain linear optimization problem is obtained and its robust counterpart is formulated. Results are presented for a two and a three dimensional test cases. In both cases, the obtained solutions are robust.

However this approach raises several limitations. The first issue appears during the approximation of constraint level curves. The way of building the secant hyperplanes from a family of points from the level curve is heuristic. In dimension two and three, a heuristic method that consists in merging closest points to build hyperplanes and then browse the level curve going through all directions, allows to construct a good approximation. It may be more difficult in higher dimension. A further step in the study would be to find a general methodology to build a piecewise

Table 6: Number of calls to the aircraft design process.

\begin{tabular}{lcc}
\hline & Deterministic Optimization with SQP & Robust Optimization \\
\hline $2 \mathrm{D}$ case & 25 & 160 \\
3D case & 55 & 389 \\
\hline
\end{tabular}


affine approximation of a level curve whatever the design space dimension is. The second issue is in the case when one only has uncertainties about model inputs. The choice of the propagation method to build the uncertain set on output is important. The need of being conservative makes fundamental the accuracy of the output error interval. An underestimation of this interval could lead to non-robust solutions.

\section{References}

Badufle, Céline. 2007. "Définition conceptuelle d'avions: vers une optimisation multi-objectif, robuste et incertaine." Ph.D. thesis. University of Toulouse III - Paul Sabatier.

Ben-Tal, Aharon, Laurent El Ghaoui, and Arkadi Nemirovski. 2009. "Robust optimization." Princeton University Press .

Ben-Tal, Aharon, and Arkadi Nemirovski. 2001. "Lectures on modern convex optimization: analysis, algorithms, and engineering applications." MPS-SIAM Series on Optimization 2.

Beyer, Hans-Georg, and Bernhard Sendhoff. 2007. "Robust optimization-a comprehensive survey." Computer methods in applied mechanics and engineering 196 (33): 3190-3218.

Birman, Jessie. 2013. "Uncertainty quantification and propagation in Conceptual Aircraft Design: from deterministic optimization to chance constrained optimization." Ph.D. thesis. University of Toulouse III - Paul Sabatier.

Bonnans, Joseph-Frédéric, Jean Charles Gilbert, Claude Lemaréchal, and Claudia A Sagastizábal. 2006. "Numerical optimization: theoretical and practical aspects." Springer .

Bury, Karl. 1999. "Statistical Distributions in Engineering." Cambridge University Press Cambridge Books Online.

Clément, Joël. 2009. "Optimisation multidisciplinaire: étude théorique et application à la conception des avions en phase d'avant projet." Ph.D. thesis. ISAE Toulouse.

Duvigneau, Régis, Massimiliano Martinelli, Praveen Chandrashekarappa, et al. 2010. "Uncertainty Quantification for Robust Design." Multidisciplinary Design Optimization in Computational Mechanics .

Ellison, Stephen LR, Matthias Rosslein, Alex Williams, et al. 2012. Quantifying uncertainty in analytical measurement. Eurachem/CITAC Guide CG4.

Geletu, Abebe, Michael Klöppel, Hui Zhang, and Pu Li. 2013. "Advances and applications of chance-constrained approaches to systems optimisation under uncertainty." International Journal of Systems Science 44 (7): 1209-1232.

Henrion, René. 2006. "Some remarks on value-at-risk optimization." International Journal of Management Science and Engineering Management 1 (2): 111-118.

Jaeger, Laure. 2013. "Optimisation multidisciplinaire sous incertitude en phase conceptuelle avion." Ph.D. thesis. University of Toulouse III - Paul Sabatier.

Jin, Ruichen, Xiaoping Du, and Wei Chen. 2003. "The use of metamodeling techniques for optimization under uncertainty." Structural and Multidisciplinary Optimization 25 (2): 99-116.

Karmarkar, Narendra. 1984. "A new polynomial-time algorithm for linear programming." Combinatorica, Springer 373-395.

Lee, Tae Won, and Byung Man Kwak. 1987. "A reliability-based optimal design using advanced first order second moment method." Journal of Structural Mechanics 15 (4): 523-542. 
Morgan, Millett G, and Max Henrion. 1990. Uncertainty: A Guide to Dealing with Uncertainty in Quantitative Risk and Policy Analysis. Cambridge University Press.

Nilsen, Thomas, and Terje Aven. 2003. "Models and model uncertainty in the context of risk analysis." Reliability Engineering 83 System Safety 79 (3): 309-317.

Padulo, Mattia, Michele Sergio Campobasso, and Marin D. Guenov. 2011. "Novel Uncertainty Propagation Method for Robust Aerodynamic Design." AIAA Journal 49 (3): 530-543.

Padulo, Mattia, Michele Sergio Campobasso, Marin Dimitrov Guenov, et al. 2007. "Comparative analysis of uncertainty propagation methods for robust engineering design." Guidelines for a Decision Support Method Adapted to NPD Processes .

Padulo, Mattia, and Meng-Sing Liou. 2013. "A MinMax Framework for Robust Design Optimization." $10^{\text {th }}$ World congress on structural and multidisciplinary optimization, Orlando (FL)

Pearson, Karl. 1936. "Method of moments and method of maximum likelihood." Biometrika 34-59.

Prigent, Sylvain, Mathieu Belleville, Thierry Druot, Aude Rondepierre, and Pierre Maréchal. 2013. "Chance constrained business case of a three-engines hybrid aircraft." $10^{\text {th }}$ World congress on structural and multidisciplinary optimization, Orlando (FL).

Scilab Enterprises. 2012. Scilab: Le logiciel open source gratuit de calcul numérique. Scilab Enterprises. Orsay, France. http://www.scilab.org.

Taguchi, G. 1978. "Performance analysis design." The international journal of production research 16 (6): $521-530$.

Tichỳ, Milík. 1994. "First-order third-moment reliability method." Structural Safety 16 (3): 189200.

Wendt, Moritz, Pu Li, and Günter Wozny. 2002. "Nonlinear chance-constrained process optimization under uncertainty." Industrial \& engineering chemistry research 41 (15): 3621-3629.

Zhou, Jian L, and Andre L Tits. 1992. "User's Guide for FSQP Version 3.0 c: A FORTRAN Code for Solving Constrained Nonlinear (Minimax) Optimization Problems, Generating Iterates Satisfying All Inequality and Linear Constraints." Institute for Systems Research Technical Reports .

\section{A}

This appendix is dedicated to the conversion of the uncertain optimization problem to its equivalent robust counterpart. Let define: $P_{k}=\frac{\zeta_{k}^{s u p}-\zeta_{k}^{i n f}}{2}$. The following equation are equivalent:

$$
\begin{aligned}
& \forall(A, b, c, d) \in \mathcal{U}:\left\{\begin{array}{l}
\forall i=1, \ldots, m, a_{i}^{\top} X \leq b_{i}, \\
c^{\top} X-t \leq-d,
\end{array}\right. \\
& \Leftrightarrow \forall(A, b, c, d) \in \mathcal{U}:\left\{\begin{array}{l}
\forall i=1, \ldots, m, \sum_{j=1}^{n} a_{i, j} \cdot x_{j} \leq b_{i}, \\
\sum_{j=1}^{n} c_{j} \cdot x_{j}-t \leq-d,
\end{array}\right. \\
& \Leftrightarrow \forall \xi \in[-1,1]^{L}:\left\{\begin{array}{l}
\forall i=1, \ldots, m, \sum_{j=1}^{n} \hat{a}_{i, j}^{(0)} \cdot x_{j}+\sum_{j=1}^{n} P_{i, j}^{(a)} \cdot \xi_{i, j} \cdot x_{j} \leq \hat{b}_{i}^{(0)}+P_{i}^{(b)} \cdot \xi_{b}, \\
\sum_{j=1}^{n} \hat{c}_{j}^{(0)} \cdot x_{j}+\sum_{j=1}^{n} P_{j}^{(c)} \cdot \xi_{j} \cdot x_{j}-t \leq-\hat{d}^{(0)}-P^{(d)} \cdot \xi_{d},
\end{array}\right. \\
& \Leftrightarrow \forall \xi \in[-1,1]^{L}:\left\{\begin{array}{l}
\forall i=1, \ldots, m, \sum_{j=1}^{n} \xi_{i, j} \cdot P_{i, j}^{(a)} x_{j}-\xi_{b} \cdot P_{i}^{(b)} \leq \hat{B}_{i}^{(0)}, \\
\xi_{d} \cdot P^{(d)}+\sum_{j=1}^{n} \xi_{j} \cdot P_{j}^{(c)} x_{j} \leq \hat{D}^{(0)},
\end{array}\right.
\end{aligned}
$$




$$
\begin{aligned}
& \Leftrightarrow\left\{\begin{array}{l}
\forall i=1, \ldots, m, \sum_{j=1}^{n} \max _{-1 \leq \xi_{i, j} \leq 1}\left[\xi_{i, j} \cdot P_{i, j}^{(a)} x_{j}\right]-\min _{-1 \leq \xi_{b} \leq 1}\left[\xi_{b} \cdot P_{i}^{(b)}\right] \leq \hat{B}_{i}^{(0)}, \\
\max _{-1 \leq \xi_{d} \leq 1}\left[\xi_{d} \cdot P^{(d)}\right]+\sum_{j=1}^{n} \max _{-1 \leq \xi_{j} \leq 1}\left[\xi_{j} \cdot P_{j}^{(c)} x_{j}\right] \leq \hat{D}^{(0)},
\end{array}\right. \\
& \Leftrightarrow\left\{\begin{array}{l}
\forall i=1, \ldots, m, \sum_{j=1}^{n} P_{i, j}^{(a)}\left|x_{j}\right|+P_{i}^{(b)} \leq \hat{B}_{j}^{(0)}, \\
P^{(d)}+\sum_{j=1}^{n} P_{j}^{(c)}\left|x_{j}\right| \leq \hat{D}^{(0)},
\end{array}\right. \\
& \Leftrightarrow \exists u \in \mathbb{R}^{n}:\left\{\begin{array}{l}
\forall j=1, \ldots, n,\left|x_{j}\right| \leq u_{j}, \\
\forall i=1, \ldots, m, \sum_{j=1}^{n} P_{i, j}^{(a)} u_{j}+P_{i}^{(b)} \leq \hat{B}_{i}^{(0)}, \\
\sum_{j=1}^{n} P_{j}^{(c)} u_{j}+P^{(d)} \leq \hat{D}^{(0)},
\end{array}\right. \\
& \Leftrightarrow\left\{\begin{array}{l}
\forall j=1, \ldots, n, x_{j}-u_{j} \leq 0, \\
\forall j=1, \ldots, n,-x_{j}-u_{j} \leq 0, \\
\forall i=1, \ldots, m, \sum_{j=1}^{n} P_{i, j}^{(a)} u_{j}+P_{i}^{(b)} \leq \hat{B}_{i}^{(0)}, \\
\sum_{j=1}^{n} P_{j}^{(c)} u_{j}+P^{(d)} \leq \hat{D}^{(0)}
\end{array}\right. \\
& \Leftrightarrow\left\{\begin{array}{l}
\forall j=1, \ldots, n, x_{j}-u_{j} \leq 0, \\
\forall j=1, \ldots, n,-x_{j}-u_{j} \leq 0, \\
\forall i=1, \ldots, m, \sum_{j=1}^{n} P_{i, j}^{(a)} u_{j}+\sum_{j=1}^{n} \hat{a}_{i, j}^{(0)} \cdot x_{j}+P_{i}^{(b)}-\hat{b}_{i}^{(0)} \leq 0, \\
\sum_{j=1}^{n} P_{j}^{(c)} u_{j}+\sum_{j=1}^{n} \hat{c}_{j}^{(0)} \cdot x_{j}-t+P^{(d)}+\hat{d}^{(0)} \leq 0,
\end{array}\right. \\
& \Leftrightarrow A \tilde{X} \leq b \text {. }
\end{aligned}
$$

In the above equations: $\tilde{\mathbf{X}}=\left(X^{\top}, t, \mathbf{u}^{\top}\right)^{\top}$, and:

$$
\begin{aligned}
& \forall i=1, \ldots, m, \hat{B}_{i}^{(0)}=\hat{b}_{i}^{(0)}-\sum_{j=1}^{n} \hat{a}_{i, j}^{(0)} \cdot x_{j}, \\
& \hat{D}^{(0)}=-\hat{d}^{(0)}-\sum_{j=1}^{n} \hat{c}_{j}^{(0)} \cdot x_{j}+t, \\
& \mathbf{C}^{\prime}=(0, \ldots, 0,1,0, \ldots, 0)^{\top} \in \mathbb{R}^{2 n+1}, \\
& \mathbf{A}=\left(\begin{array}{ccc}
I_{n} & 0_{n} & -I_{n} \\
-I_{n} & 0_{n} & I_{n} \\
\hat{A^{(0)}} & 0_{m} & P^{(a)} \\
\hat{\mathbf{C}^{(\mathbf{0})}} & -1 & \mathbf{P}^{(\mathbf{c})}
\end{array}\right) \in \mathbb{R}^{(2 n+m+1) \times(2 n+1)}, \\
& \mathbf{b}=\left(0_{1 \times 2 n}, \hat{\mathbf{b}}^{(\mathbf{0})}-\mathbf{P}^{(\mathbf{b})},-P^{(d)}-\hat{d}^{(0)}\right)^{\top} \in \mathbb{R}^{(2 n+m+1)}, \\
& \hat{\mathbf{A}^{(\mathbf{0})}}=\left(\begin{array}{ccc}
\hat{a}_{1,1}^{(0)} & \ldots & \hat{a}_{1, n}^{(0)} \\
\vdots & \ldots & \vdots \\
\hat{a}_{m, 1}^{(0)} & \ldots & \hat{a}_{m, n}^{(0)}
\end{array}\right), \mathbf{P}^{(\mathbf{a})}=\left(\begin{array}{ccc}
P_{1,1}^{(a)} & \ldots & P_{1, n}^{(a)} \\
\vdots & \ldots & \vdots \\
P_{m, 1}^{(a)} & \ldots & P_{m, n}^{(a)}
\end{array}\right), \\
& \hat{\mathbf{C}^{(\mathbf{0})}}=\left(\hat{c}_{1}^{(0)}, \ldots, \hat{c}_{n}^{(0)}\right), \mathbf{P}^{(\mathbf{c})}=\left(P_{1}^{(c)}, \ldots, P_{n}^{(c)}\right)^{\top}, \\
& \mathbf{b}^{\hat{(0)}}=\left(\hat{b}_{1}^{(0)}, \ldots, \hat{b}_{m}^{(0)}\right), \mathbf{P}^{(\mathbf{b})}=\left(P_{1}^{(b)}, \ldots, P_{m}^{(b)}\right) \text {. }
\end{aligned}
$$

